\title{
Photoacoustic imaging probe for detecting lymph nodes and spreading of cancer at various depths
}

\author{
Yong-Jae Lee \\ Eun-Ju Jeong \\ Hyun-Woo Song \\ Chang-Geun Ahn \\ Hyung Wook Noh \\ Joo Yong Sim \\ Dong Hoon Song \\ Min Yong Jeon \\ Susung Lee \\ Heewon Kim \\ Meihua Zhang \\ Bong Kyu Kim
}




\title{
Photoacoustic imaging probe for detecting lymph nodes and spreading of cancer at various depths
}

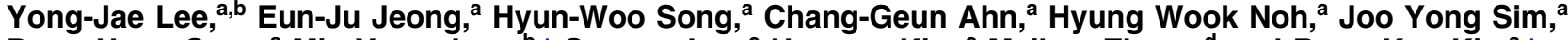 \\ Dong Hoon Song, ${ }^{a}$ Min Yong Jeon, ${ }^{b, *}$ Susung Lee, ${ }^{c}$ Heewon Kim, ${ }^{c}$ Meihua Zhang, ${ }^{d}$ and Bong Kyu Kim ${ }^{a, *}$ \\ aElectronics and Telecommunications Research Institute, Diagnostic Device Research Section, Daejeon, Republic of Korea \\ ${ }^{\mathrm{b}}$ Chungnam National University, Department of Physics, Daejeon, Republic of Korea \\ ${ }^{c}$ Alpinion Medical System Co., Ltd., Probe Development Team, Seoul, Republic of Korea \\ ${ }^{d}$ Seoul National University Hospital, Radiology of Breast Care Center, Seoul, Republic of Korea
}

\begin{abstract}
We propose a compact and easy to use photoacoustic imaging (PAl) probe structure using a single strand of optical fiber and a beam combiner doubly reflecting acoustic waves for convenient detection of lymph nodes and cancers. Conventional PAI probes have difficulty detecting lymph nodes just beneath the skin or simultaneously investigating lymph nodes located in shallow as well as deep regions from skin without any supplementary material because the light and acoustic beams are intersecting obliquely in the probe. To overcome the limitations and improve their convenience, we propose a probe structure in which the illuminated light beam axis coincides with the axis of the ultrasound. The developed PAI probe was able to simultaneously achieve a wide range of images positioned from shallow to deep regions without the use of any supplementary material. Moreover, the proposed probe had low transmission losses for the light and acoustic beams. Therefore, the proposed PAI probe will be useful to easily detect lymph nodes and cancers in real clinical fields. $\odot 2017$ Society of Photo-Optical Instrumentation Engineers (SPIE) [DOI: 10.1117/1.JBO.22.9.091513]
\end{abstract}

Keywords: photoacoustic; ultrasound; probe.

Paper 160825SSR received Dec. 2, 2016; accepted for publication Apr. 5, 2017; published online Apr. 25, 2017.

\section{Introduction}

Photoacoustic imaging (PAI) can provide in vivo functional and molecular imaging by using ultrasonic waves generated by pulsed laser light signals. ${ }^{1}$ It has the advantage of being able to easily identify the properties of the target materials such as lymph nodes, cancer cells, capillary blood vessels, and the oxygen saturation of hemoglobin. ${ }^{2}$ In addition, the PAI imaging system can achieve large-depth imaging like diffuse optical tomography ${ }^{3}$ or high-resolution imaging like optical coherence tomography. ${ }^{4}$ Therefore, PAI has great potential for future diagnostic imaging. 5

For diagnostic detection of lymph nodes and cancers, the PAI system has to be able to achieve both shallow and deep imaging because human lymph nodes are positioned at various depths. ${ }^{6}$ Additionally, the PAI system needs real-time monitoring and a compact structure in order to be useful in medical imaging diagnosis. Recently, the handheld PAI probe structure and system that can achieve deeply penetrating and real-time imaging has attracted increasing research interest.

Most developed handheld PAI probes employ an optical fiber or optical fiber bundles attached to the side of the ultrasound transducer for light beam delivery. ${ }^{7-12}$ The probes have the merits of low transmission losses of light and sound, because they do not use any combiner so that a probe with the structure can receive the largest photoacoustic signal as a function of the light intensity. ${ }^{13}$ However, the detectable imaging region depends on the angle of incident light and there is an undetectable zone, called dark-field illumination, because the illuminated light

*Address all correspondence to: Min Yong Jeon, E-mail: myjeon@cnu.ac.kr; Bong Kyu Kim, E-mail: bongkim@etri.re.kr beam is oblique with respect to the acoustic beam, and the image is obtained in the intersecting region between the illuminating light beam and receiving acoustic beam. In order to obtain images at shallow depths, the probe may require a dummy material such as a chicken breast or water. Otherwise, it is hard to simultaneously detect the images in the shallow as well as deep regions without any supplementary or dummy material. The property can result in inconveniences in real in vivo clinical applications for investigating lymph nodes spread at various depths.

To eliminate the undetectable range and expand the detectable imaging range, a bright-field PAI probe structure was reported in a previous work ${ }^{14}$ as similar to the bright-field photoacoustic microscopic structure. ${ }^{15}$ In a bright-field PAI probe, the light beam and the ultrasound beam are combined as shown in Fig. 1(c). The illuminated light beam path coincides with the ultrasound beam path in the skin detection area. Thus, it can obtain PAI in shallow and deep regions at the same time. However, this PAI probe has a wide skin contact area because the ultrasound transducer is perpendicularly positioned to the light beam delivery module, making an L-shape. With such a probe, it would be inconvenient to examine particular areas such as the clavicle and armpit in diagnosis. Moreover, general PAI probes employ optical fiber bundles to transmit high-power laser beams and irradiate wide areas. However, the probe cables composed of the optical fiber bundle and coaxial cable tend to be rigid, which prevents the PAI probes from the practical use in clinic.

This paper proposes a compact and easy to use PAI probe structure providing a bright-field illumination system. First, the proposed probe structure is designed to have a small skin contact

$1083-3668 / 2017 / \$ 25.00$ @ 2017 SPIE 


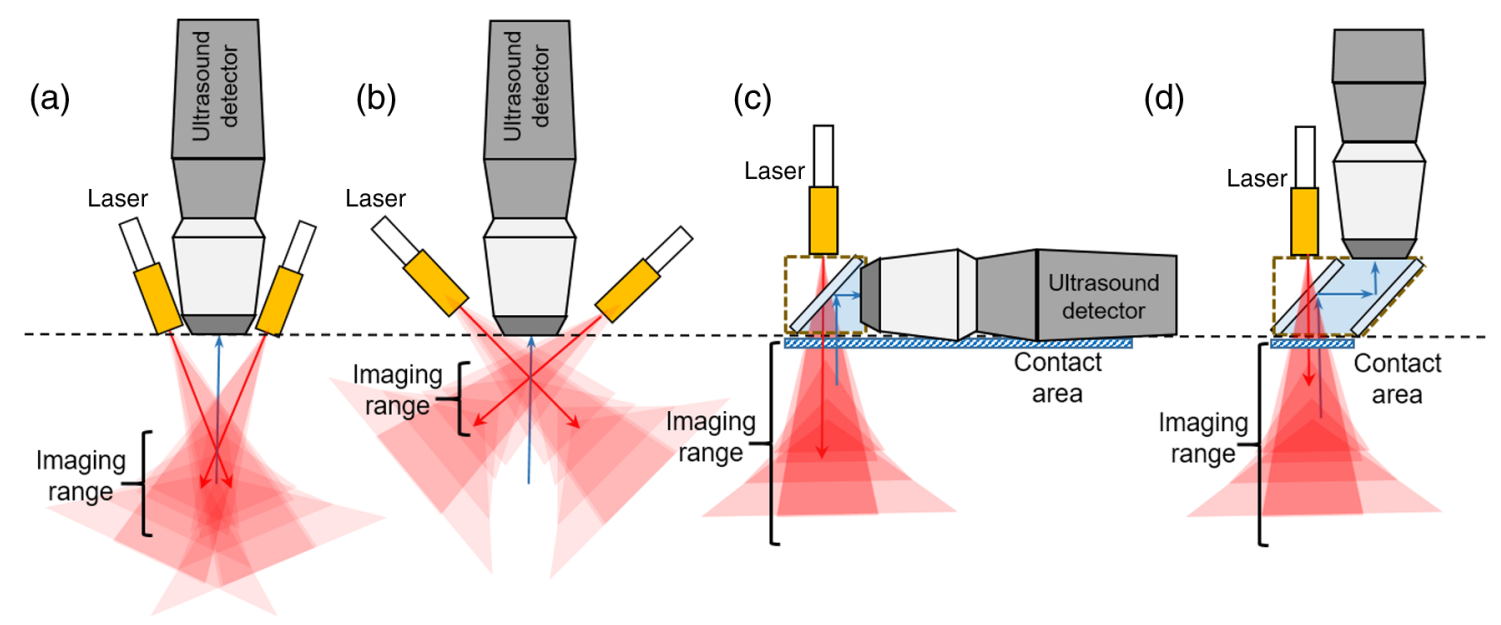

Fig. 1 Various PAI structures. (a) and (b) Dark-field illumination structures: the previous structure has an undetectable zone in which it cannot detect ultrasound imaging caused by oblique illumination. (a) Difficult to detect shallow region. (b) Difficult to detect deep region. (c) and (d) Bright-field illumination structures: to remove the dead zone and expand the imaging range. (c) Previous bright-field illumination structure that has wide skin contact area. (d) Proposed photoacoustic probe structure that has a small skin contact area.

area as an ultrasound detector is positioned parallel to the light beam delivery module [Fig. 1(d)]. This approach, similar to an optical resolution photoacoustic microscopic probe (I-shape), ${ }^{16}$ can provide a compact and handheld structure. Therefore, it becomes easy to obtain PAI in the armpit area. Second, one strand of a multimode optical fiber is used as a high-power laser transmission material instead of optical fiber bundles. This use of a single-fiber strand not only makes the cable lighter, but also increases the efficiency of light transmission compared to the bundles of multiple fibers and it still delivers a high laser power. Because of the efficient high transmission, a deeper image can be achieved with the same energy of pump laser energy. In other words, lower pump laser energy would be needed for the same characteristics; therefore, the size and cost of the light source can be reduced. Finally, the probe is designed to have a line-shaped light beam by using three cylindrical lenses for B-scan PAI and real-time PAI. We here demonstrate the proposed PAI probe and achieved images in shallow as well as deep regions simultaneously. Moreover, it was also possible to detect the lymph node of a nude mouse without any supplementary material. The developed probe was compact and easy to use, and the detection imaging range was expanded. It would be useful to detect lymph nodes spread at various depths and other tissues in the armpit area.

\section{Materials and Methods}

Figure 2 shows the schematic diagram of the proposed PAI probe, which was composed of a beam combiner, a linebeam generator, and an ultrasound array detector. On the longitudinal view, the dotted box indicates the line-beam generator, which consisted of three cylindrical lenses (order production, Teleoptics Co., Ltd., Korea) with antireflection coatings and one multimode optical fiber with an optical fiber connector. The optical pulse was emitted from the multimode optical fiber with a solid angle by the numerical aperture of the optical fiber. A convex cylindrical lens (lens 1) collimates the optical pulse beam emitted from the optical fiber in the transverse direction and another convex cylindrical lens (lens 2) widely collimates the optical pulse beam passed through lens 1 in the longitudinal direction. The other convex cylindrical lens (lens 3) focuses the collimated optical pulse beam from lens 2 in the transverse direction. Finally, the output laser beam at the end of the linebeam generator had a line-shape. The line-shaped beam irradiating the tissue after passing through a beam combiner is illustrated in the bottom dotted box of the transverse view of Fig. 2. The ultrasound generated by the line-shaped beam was double-reflected perpendicularly to the two-slide glasses (microscope slides, Marienfeld-Superior) inside the line beam and proceeded into an ultrasound detector.

The beam combiner not only aligned the light and ultrasound beams to the same plane, but also provided a compact one-body structure for easy handling because the line-beam generator was positioned parallel to the ultrasound transducer. The beam combiner was composed of two-slide glasses and was filled with distilled water. Here, filling the beam combiner with water improved the transmission characteristics by light index matching and acoustic impedance matching. The light with a 45-deg incident angle had a theoretical transmission loss of $0.97 \mathrm{~dB}$ at slide glass 1 ( $\left.n_{\text {glass }}=1.5\right)$ in the water. Moreover, the ultrasound wave with a 45-deg incident angle was totally perpendicularly reflected on the two-slide glasses in the water because the critical angle for total reflection was estimated to be $15.6 \mathrm{deg}$, which was calculated using the acoustic impedance values for the slide glass $\left[Z_{\text {glass }}=12.3 \times 10^{6} \mathrm{~kg} /\left(\mathrm{m}^{2} \mathrm{~s}\right)\right]$ and water. The transmission loss for the ultrasound wave passing through the water can be negligible because water has an ultrasound attenuation of $0.002 \mathrm{~dB} /(5 \mathrm{MHz} \mathrm{cm})$.

To prevent water leakage from the beam combiner, two plastic plates (acrylic and low-density clear plastic) were used. The output beam from the line-beam generator passed through the acrylic plate, which was coated for antireflection of the 780-nm incident light wavelength. The low-density clear plastic on the bottom of the probe was used as a probe window, and the estimated acoustic and optical transmission losses from reflection were 0.1 and $0.01 \mathrm{~dB}$, respectively. Here, the low-density clear plastic had an acoustic impedance $\left[Z_{\text {plastic }}=1.84 \times 10^{6} \mathrm{~kg} /\left(\mathrm{m}^{2} \mathrm{~s}\right)\right]$ and refractive index $\left(n_{\text {plastic }}=1.46\right)$ similar to the water. Inside the low-density clear plastic with a thickness of $1 \mathrm{~mm}$ and 


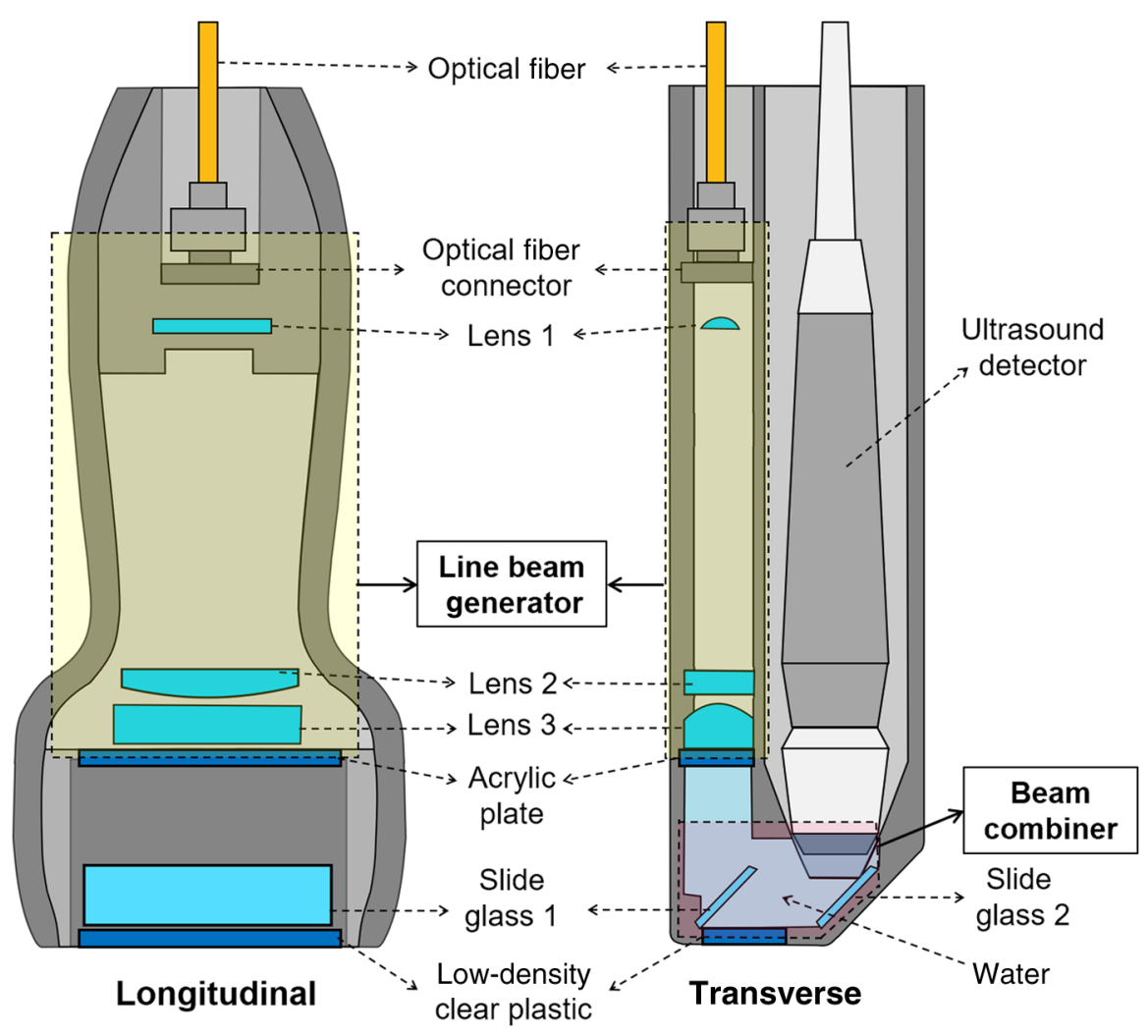

Fig. 2 Schematic of the PAI probe that has the line beam generator and the beam combiner.

acoustic attenuation coefficient of $1.1 \mathrm{~dB} /(\mathrm{MHz} \mathrm{cm})$, the ultrasound attenuation loss was estimated to be $0.55 \mathrm{~dB}$ at a frequency of $5 \mathrm{MHz}$. The total acoustic and optical transmission losses by the beam combiner and the windows were estimated to be 0.65 and $0.98 \mathrm{~dB}$, respectively.

\section{System Performance}

The casing of the PAI probe had a curved shape for convenience in medical diagnosis. The bottom face size of the PAI probe was $55 \times 25 \mathrm{~mm}^{2}$ for easy handling of the bright-field illumination PAI probes. Figure 3(a) shows a photograph of the line-shaped beam illumination $\left(20 \times 5.5 \mathrm{~mm}^{2}\right)$ emitted from the PAI probe at 780-nm wavelength, and Fig. 3(b) shows a three-dimensional (3-D) intensity profile of the line-shaped beam performance generated at the PAI probe using a beam profiler (Beamon LA, Duma Optronics Ltd.). We used a compact gain-switched Ti: sapphire laser pumped by a frequency-doubled Nd:YAG pulse laser (Ultra, Quantel Laser) operated at an output energy of $37 \mathrm{~mJ}$ with a 532-nm wavelength, $10-\mathrm{Hz}$ repetition rate, and 11-ns pulse duration. ${ }^{17}$ The pulsed laser was coupled into a connectorized multimode optical fiber cable (\#HP-2020-10A3CH$040 \mathrm{E}$, Fiberguide industries) with a $1000-\mu \mathrm{m}$ core and 0.22 numerical aperture.

The optical fiber cable was connected at the PAI probe as shown in Fig. 2. The emitted laser pulse energy at the end of the probe was $6.3 \mathrm{~mJ}$ per pulse, corresponding to a laser fluence of $5.3 \mathrm{~mJ} / \mathrm{cm}^{2}$. The total optical loss from the laser to the probe output was experimentally measured to be $2.4 \mathrm{~dB}$. Figures $3(\mathrm{c})$ and 3(d) show the intensity distributions of the line-shaped beam using a beam. The beam sizes in the transverse and longitudinal directions were 5.6 and $21 \mathrm{~mm}$, respectively. The transverse intensity distribution shows that the line-shaped optical beam was well formed by the three cylindrical lenses. Although the proposed probe utilized a single-strand optical fiber cable, it delivered sufficient optical energy to obtain PAI.

Figure 4(a) shows a schematic diagram of the PAI acquisition system using the proposed PAI probe to confirm the bright-field illumination system. An ultrasound detector (L3-8, Alpinion Medical Systems) with 128 elements and an ultrasonography machine (E-cube 9, Alpinion Medical Systems) was used to acquire the PAI. The ultrasound detector had a center frequency of $5 \mathrm{MHz}$, focal length of $35 \mathrm{~mm}$, and $6 \mathrm{~dB}$ bandwidth of $66.78 \%$.

In order to acquire the photoacoustic signals and test the detection range, we used an indocyanine green (ICG) tube containing $1 \mathrm{mg} / \mathrm{ml}$ ICG solution diluted in dimethyl sulfoxide whose absorption peak is $780 \mathrm{~nm} .{ }^{18}$ The ICG tube was $10-\mathrm{cm}$ in length and 5-mm in diameter and placed between polyvinyl chloride plastisol (PVCP) phantom with 0.25 wt. $\% \mathrm{TiO}_{2}$. The PVCP served as a phantom material because it was found to be similar enough to human breast tissues in photoacoustic properties. ${ }^{19}$ PAIs from the ICG positioned at various depths were well obtained at the various depths as shown in Fig. 4(b). These results show that our system could achieve PAI in the shallow and deep regions with a single probe and without changing its configuration.

To test the shallow PAI, we obtained images of the lymph nodes positioned immediately below the skin by using an in vivo small animal model. In order to dye the lymph nodes, the ICG solution with the concentration of $1 \mathrm{mM}(0.78 \mathrm{mg} / \mathrm{mL})$ diluted in the dimethyl sulfoxide solution was injected into a nude mouse. In the following experiments, we used another ultrasound detector (L8-17, Alpinion Medical Systems) with 128 elements. The ultrasound detector had a center frequency of 
(a)

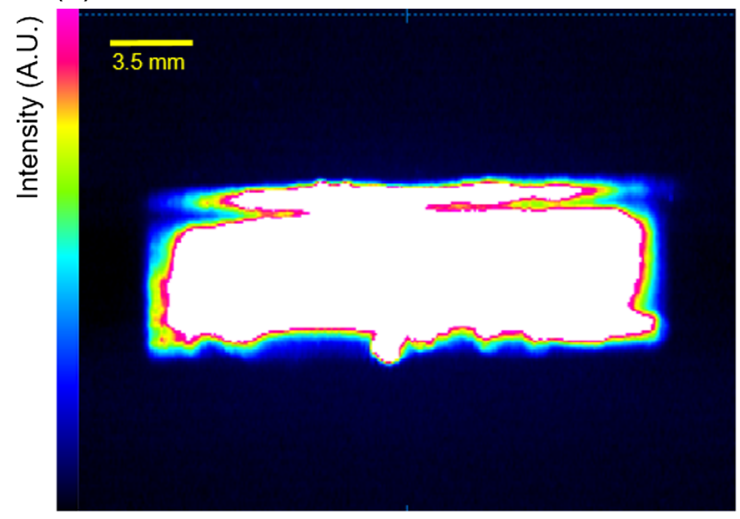

(c)

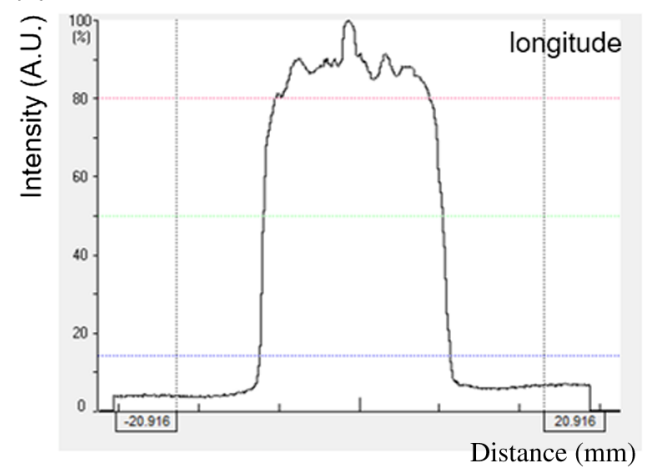

(b)

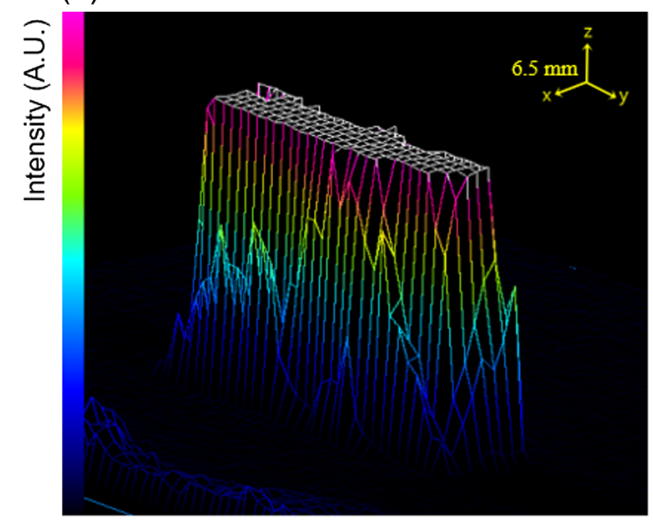

(d)

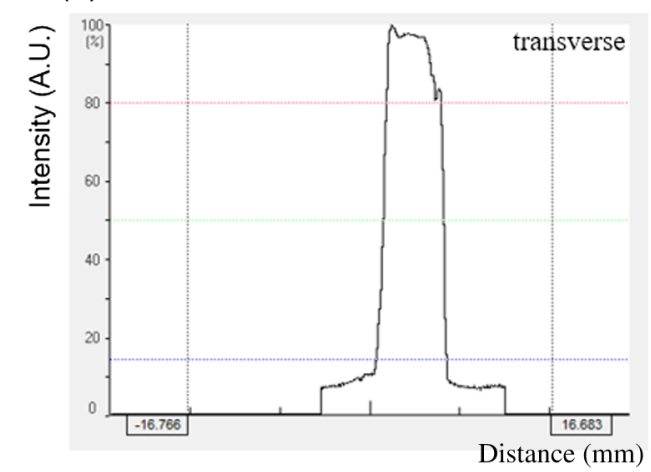

Fig. 3 Characteristics of the proposed probe using a beam profiler. (a) Two-dimensional and (b) 3-D plots of line-shaped beam intensity showing the performance of the PAI probe; (c) and (d) longitude and transverse profiles of the beam intensity distribution.

$10 \mathrm{MHz}$, focal length of $20 \mathrm{~mm}$, and 6-dB bandwidth of $58.95 \%$.

Figure 5(a) shows the imaging of lymph nodes, which contains the photoacoustic (color) and ultrasound (monochrome) imaging of two dyed brachial and axillary lymph nodes of the mouse. The detected image depths were measured to be $0.5 \mathrm{~mm}$ (brachial) and $0.7 \mathrm{~mm}$ (axillary) from the mouse skin, respectively, and both sizes of the two lymph nodes were $3.0 \mathrm{~mm} \times$ $1.5 \mathrm{~mm}$.
When optical fiber bundles are attached to the side of an ultrasound receiver, such a PAI probe generally requires either imaging in a bath filled with water or being supplemented with chicken breast tissue as a dummy material to enable PAI of substances just beneath the skin. On the contrary, the proposed probe obtained the PAI by using only an ultrasound gel between the PAI probe and mouse without any supplementary material as shown in Fig. 5(b). To check the depth of the brachial lymph nodes, we made a 5- to 8-mm incision as shown in Fig. 5(c). (a) Ultrasonography

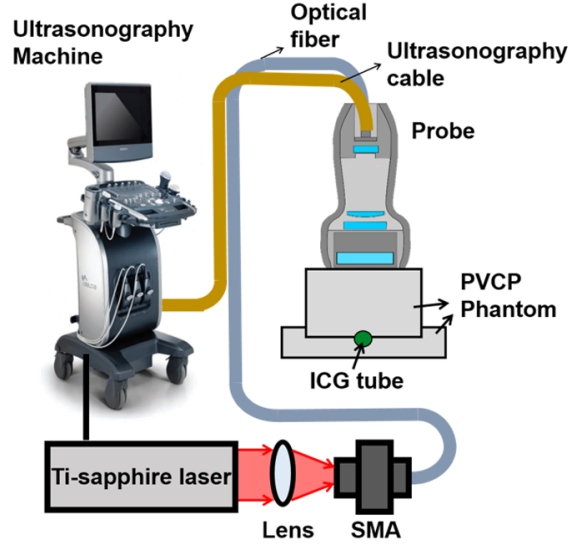

(b) (mm)

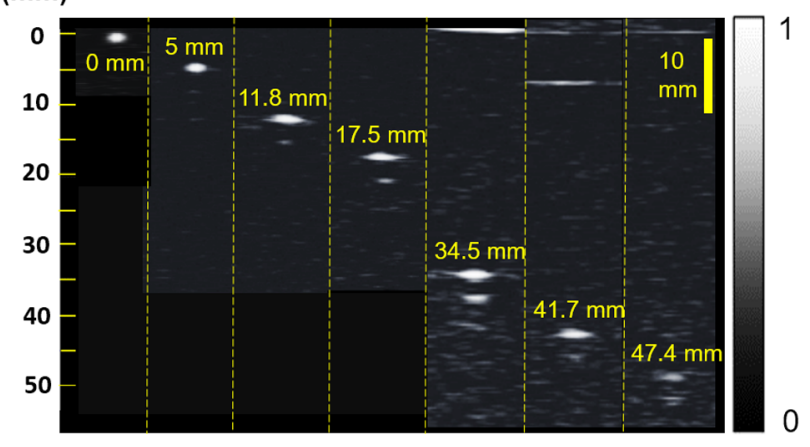

Fig. 4 (a) Schematic of the proposed system to acquire PAI that can image immediately below the window of the PAI probe and (b) a merged image of each ICG image from the PAI system at various depths in the PVCP phantom. 
(a)

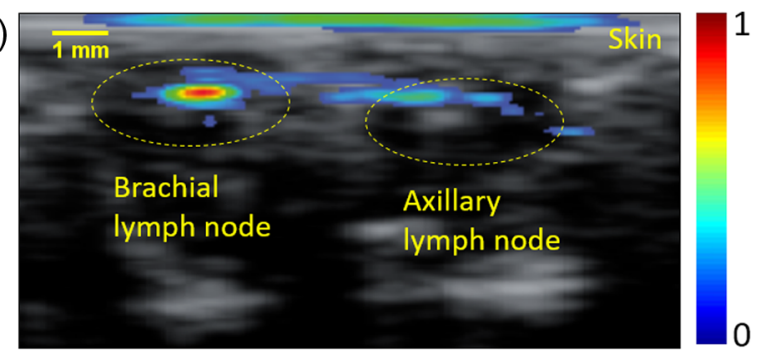

(b)

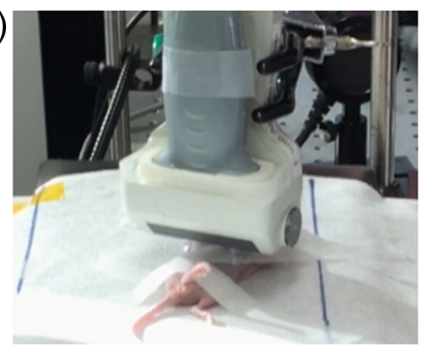

(c)

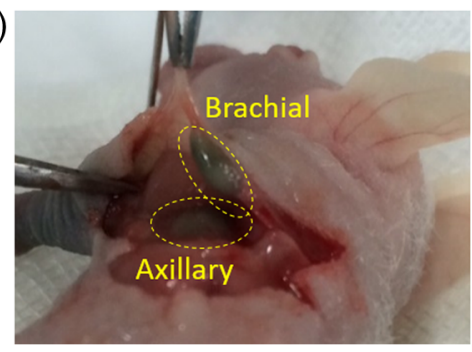

(d)

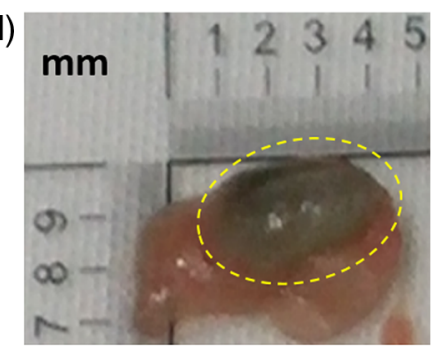

Fig. 5 (a) Imaging of lymph nodes that contain the photoacoustic (color) and ultrasound (monochrome) imaging of two dyed brachial and axillary lymph nodes of the mouse, (b) nude mouse experiment view without any material, (c) dissection imaging, and (d) extracted lymph node.

After the incision, one extracted brachial lymph node was located $1 \mathrm{~mm}$ below the skin and the size was $3.2 \mathrm{~mm} \times 1.6 \mathrm{~mm}$ as shown in Fig. 5(d). The depth and size of the lymph node detected by the proposed probe agreed well with the measured result from the dissection. Therefore, we could obtain the PAI of a lymph node existing in the shallow region beneath the skin and demonstrate that the minimum detecting depth was less than $1 \mathrm{~mm}$.

All experimental animal procedures were approved by the Institute Animal Care and Use Committee (IACUC) of Seoul National University Hospital Biomedical Research [13-0219C3A0(1)], and all experiments of imaging acquisition were performed at the Electronics and Telecommunications Research Institute.

\section{Discussion and Conclusion}

We have proposed and developed a compact PAI probe that is easy to handle because one strand of optical fiber cable was used instead of fiber bundles. Moreover, the laser output at the probe window had a line-shaped beam shape created by three cylindrical lenses to be suitable for the B-scan PAI, and the illuminated light beam path coincided with the ultrasound beam path. The developed probe obtained PAIs in the shallow as well as deep regions at the same time. However, our PAI system was not optimized for a PAI system and we will improve the PAI system using a synthetic algorithm in the future work. ${ }^{20}$

In the small animal experiment, the lymph nodes near the skin could be detected without the need for any supplementary material. The proposed compact PAI probe will be convenient and easy to use in medical diagnostics because it does not need any supplementary material. We believe that our proposed PAI probe will have a significant effect on future clinical medical diagnostics.

\section{Disclosures}

No conflicts of interest, financial or otherwise, are declared by the authors.

\section{Acknowledgments}

This research was supported by the Internal Research Program of Electronics and Telecommunications Research Institute (Grant No. 16ZC1710) and The Leading Human Resource Training Program of Regional Neo Industry through the National Research Foundation of Korea (NRF) funded by the Ministry of Science, ICT, and Future Planning (NRF-2016H1D5A1909597).

\section{References}

1. A. G. Bell, "On the production and reproduction of sound by light," Am. J. Sci. 20(118), 305-324 (1880).

2. M. Xu and L. V. Wang, "Photoacoustic imaging in biomedicine," Rev. Sci. Instrum. 77(4), 041101 (2006).

3. B. W. Zeff et al., "Retinotopic mapping of adult human visual cortex with high-density diffuse optical tomography," Proc. Natl. Acad. Sci. U. S. A. 104(29), 12169-12174 (2007).

4. D. Huang et al., "Optical coherence tomography," Science 254, 11781181 (1991).

5. J. Xia, J. Yao, and L. H. V. Wang, "Photoacoustic tomography: principles and advances (invited review)," Prog. Electromagn. Waves 147, $1-22$ (2014).

6. G. C. Bentel et al., "Variability of the depth of supraclavicular and axillary lymph nodes in patients with breast cancer: is a posterior axillary boost field necessary?" Int. J. Radiat. Oncol. Biol. Phys. 47(3), 755-758 (2000).

7. P. Ephrat et al., "White paper: imaging of murine tumors using the system," VisualSonics, http://www.visualsonics.com/sites/default/files/WP_ 2100_Cb_Photoacoustic_Imaging.pdf.

8. J. Kang et al., "Ex vivo estimation of photoacoustic imaging for detecting thyroid microcalcifications," PLoS One 9(11), e113358 (2014).

9. C. Kim et al., "Deeply penetrating in vivo photoacoustic imaging using a clinical ultrasound array system," Biomed. Opt. Express 1(1), 278 (2010).

10. J. J. Niederhauser et al., "Combined ultrasound and optoacoustic system for real-time high-contrast vascular imaging in vivo," IEEE Trans. Med. Imaging 24(4), 436-440 (2005).

11. D. W. Yang et al., "Integrative prototype B-scan photoacoustic tomography system based on a novel hybridized scanning head," Appl. Phys. Lett. 88, 174101 (2006).

12. R. G. M. Kolkman et al., "Real-time in vivo photoacoustic and ultrasound imaging," J. Biomed. Opt. 13(5), 050510 (2008). 
13. G. Wang et al., "Simulation of light delivery for photoacoustic breast imaging using the handheld probe," Chin. Opt. Lett. 12(5), 051703 (2014).

14. L. G. Montilla et al., "Real-time photoacoustic and ultrasound imaging: a simple solution for clinical ultrasound systems with linear arrays," Phys. Med. Biol. 58(1), N1-N12 (2013).

15. S. Hu, K. Maslov, and L. V. Wang, "In vivo functional chronic imaging of a small animal model using optical-resolution photoacoustic microscopy," Med. Phys. Lett. 36(6), 2320-2323 (2009).

16. S. Hu, K. Maslov, and L. V. Wang, "Second-generation optical-resolution photoacoustic microscopy with improved sensitivity and speed," Opt. Lett. 36(7), 1134-1136 (2011).

17. J. Lee et al., "Gain-switched Ti: sapphire laser-based photoacoustic imaging," Appl. Opt. 55(20), 5419-5422 (2016).

18. D. C. J. Rezende, L. D. Boni, and C. R. Mendonça, "Dynamics nonlinear optical properties in indocyanine green solutions," in Annals of Optics XXIX ENFMC, Sociedade Brasileira de Física (SBF), Brazil (2006).

19. E. J. Jeong et al., "Fabrication and characterization of PVCP human breast tissue-mimicking phantom for photoacoustic imaging," BioChip J. 11(1), 67-75 (2017).

20. C.-K. Liao, M.-L. Li, and P.-C. Li, "Optoacoustic imaging with synthetic aperture focusing and coherence weighting," Opt. Lett. 29(21), 2506-2508 (2004).

Yong-Jae Lee received his BS degree in physics from Chungnam National University, Daejeon, Korea, in 2012. He is currently pursuing his MS and PhD degrees at Chungnam National University and the Electronics and Telecommunications Research Institute, Daejeon, Korea. His research interests include optical coherence tomography and photoacoustic imaging systems.

Eun-Ju Jeong received his BS and MS degrees in biochemistry from Gyeongsang National University. She joined the Electronics and Telecommunications Research Institute (ETRI), Daejeon, Korea, and is currently a senior researcher of Medical Device Research Section. Her research experiences are in the area of biomedical optics, and biomedical optical systems. Her current research focuses are medical imaging systems, u-healthcare, and optical sensor systems and devices.

Hyun-Woo Song received his $\mathrm{PhD}$ in semiconductor optics from Korea Advanced Institute of Science and Technology (KAIST) in 1999. He joined ETRI, Daejeon, Korea, and is currently a principal researcher of the Medical Device Research Section. His research experiences are in the area of biomedical optics, biomedical optical systems, and semiconductor optical devices. His current research foci are medical tomographic imaging modules and semiconductor optical sources for optical coherence tomography and photoacoustic tomography.

Chang-Geun Ahn received his MS and PhD degrees from the Department of Electrical and Electronic Engineering in Pohang University of Science and Technology (POSTECH), Pohang, Republic of Korea in 1996 and 2000, respectively. From 2000 to 2013, he worked for the same university and California Institute of Technology as a postdoc. Since 2013, he has been with ETRI, where he is now a principal research engineer. His research interests include electronic devices, biosensors, and healthcare devices.

Hyung Wook Noh received his BS and MS degrees from Yonsei University, Korea, in 2008 and 2010, respectively. He joined ETRI, Daejeon, Korea, and is currently a researcher of the Medical Device
Research Section, and his research interests include medical imaging system, u-healthcare, and biosignal sensor systems and devices.

Joo Yong Sim received his BS degree in mechanical and aerospace engineering from Seoul National University and his MS and PhD degrees in mechanical engineering from Stanford University. Since graduating with his $\mathrm{PhD}$, he has been working as a researcher at the Electronics and Telecommunications Research Institute. His research is primarily in the area of biomedical engineering and biophysics focusing on the development of biomedical sensors using microelectromechanical systems, microfabricated devices, biophotonics, and advanced microscopy techniques.

Dong Hoon Song received his BS degree in applied chemical engineering from Yeungnam University, Korea, in 2003, his MS degree in information and communication engineering in 2007, and his PhD in physics in 2012 from GIST, Korea. In 2012, he joined the Electronics and Telecommunications Research Institute, Korea. His current interests are the technology development of ultrafast lasers and their applications in bioimaging.

Min Yong Jeon received his BS degree from Han Yang University, Seoul, Korea, in 1988, and his MS and PhD degrees from KAIST, Daejeon, Korea, in 1990 and 1994, respectively, all in physics. He has been with Chungnam National University, Daejeon, Korea, as a professor of physics since May 2003. His current research focuses on optical coherence tomography, wavelength swept lasers, optical fiber sensors, and optical terahertz wave generation.

Susung Lee received his $\mathrm{PhD}$ in sensor and display engineering from Kyungbook National University, Korea, in 2008 . He is currently a principal research engineer at R\&D of Alpinion Medical Systems, Seoul, Korea. Before joining Alpinion in 2009, he worked in the Department of Medical Ultrasound Transducer, Siemens Ultrasound System, Korea. His current research interests include the development of valuable innovative medical ultrasound transducers with new architectures of acoustic stacks, new active and passive materials, new fabrication process, and handle electronics.

Heewon Kim received his BS degree in mechanical design engineering from Kumoh National Institute of Technology, Gumi, Korea, in 2001, and his MS degree in mechanical engineering from Kyungpook National University, Daegu, Korea, in 2003. From 2003 to 2009, he was a principal R\&D engineer at the Siemens Ultrasound Division, Pohang, Korea. He joined Alpinion Medical Systems, Seoul, Korea, where he is currently a senior principal engineer of R\&D Division. His research interests are in acoustic designing and the development of medical ultrasound transducers.

Meihua Zhang received her BS and MS degrees in medicine from Yanbian University, China, in 2006 and 2009, respectively. She is currently pursuing her MD and PhD degrees at Seoul National University, Seoul, Korea. From 2009 to 2013, she was with Yanbian Women Hospital, China. Her research interests include ultrasound imaging and photoacoustic imaging.

Bong Kyu Kim received his BS degree in physics from Hanyang University, Korea, in 1989, and his MS and PhD degrees in physics from KAIST, Korea, in 1992 and 1996. From 1996 to 1999, he was with the Photonics Research Center, KIST, Korea. He is currently a section leader at the Diagnostic Device Research Section, ETRI, Daejeon, Korea. His research interests include point of care testing (POCT), medical imaging system, u-healthcare, and optical sensor systems and devices. 